\title{
Applied Artificial Intelligence in 3D-game (HYSTERIA) using UNREAL ENGINE4
}

\author{
MuhammadMuzammul ${ }^{1}$, \\ Muhammad Awais ${ }^{2}$, Muhammad \\ Umer ghani ${ }^{3}$ \\ Department of software engineering \\ Government College University \\ Faisalabad, Pakistan
}

\author{
Muhammad Imran Manzoor ${ }^{4}$ \\ Department of Computer \\ Science \& IT \\ University of Lahore \\ PakPattan Campus
}

\author{
Muhammad Kashif ${ }^{5}$, Muhammad \\ Yahya Saeed $^{6}$ \\ Department of software engineering \\ Government College University \\ Faisalabad, Pakistan
}

\begin{abstract}
Game development industry spreading it roots at wider level. With the advancements in gaming technologies industries adopted latest trends for developing modern games. Artificial intelligence (AI) with programming provided countless support for latest technology adoption in game industry. This paper aims to highlight some major points of our research "Creation of third person shooter game in unreal engine 4". We discussed how we can use one of the most powerful current generation game engines in an attempt to create our own game "Hysteria". Endeavoring used to replicate the process of the major game production cycle .It is used by modern gaming industries. We attempted it to create an action adventure shooting game by creating its own original storyline. The game Hysteriaisplayedfromathirdperson perspective in which the player must go through multipleenvironmentsfightinghordesof enemies and try to reach the end of level. Depending on the difficulty level that the player sets, there will be the number of enemies and their fighting intensity. The game has been developed but running at initial stages; further enhancement will be required to give it a much professional impression so that in near future it could be successfully commercialized.
\end{abstract}

Keywords-Applied AI; UNREAL ENGIN 4; technology adoption

\section{INTRODUCTION}

The video game industry is one of the most commercially successful extensions of the computer science industry. With over billion video game enthusiasts around the world, the video game industry produces annual revenue in billions of dollar each year. Witheachgamingtitles ranging from console titles of Xbox one and Play Station4 to the free android games, grossing over millions of dollars, video game industryare at epitome of its commercializationat is still evolving year by year.Keeping in mind, our project aim to produce our own game title which will be available for commercialization in the near future. Consideringthe countrieswhich considered as key players in the video game industry such as China,JapanandAmerica, theyallhavemanynumbersof video developmentstudios andpublishingfirms who follow a certaindevelopment process of video games. Whilethe process may be somewhatsimilar to the typical software development process, it is different considering aspects of the different categories of video game. Since our game title falls into the category of thirdperson action shooter, aspects such as characterdesign, environment design and artificial design are to be considered.With studyingthe development process of the video game industry, a core game engine is required which provides a basic skeleton structure to the game body, with arrangement of different assets sequentially, it provides a linear game play experience in an exe.They all have large number of video development studios and publishing firms which follow a certain development process of video games. While the process may be somewhat similar to the typical software development process, it is different considering aspects of the different categories of video game. Since our game title falls into the category of third person action shooter, aspects such as character design, environment design and artificial design are to be considered.

With studyingthe development process of the video game industry, a core game engine is required which provides a basic skeleton structure to the game body,with arrangement of different assetssequentially,it provides a linear game play experience in an exe file of the development mode. The overall system design objective is to lay down the basic structure using the most powerful game engine of theindustryunrealengine 4 anduse variousothersoftware to createa sequentiallyincrementingAAA video game title.The game will be available to play on PC, PS4 and Xbox one, and thus will be a current generation game title running on advance recent consoles.The actual game play will be in third person shooting covering structure and with its original storyline; it will be available for commercialization upon further development. The scope of this project is to design and develop a game title using Epic games most recent incarnation of its unreal engine game engines series, unreal engine 4 . The game title will be a third person action adventure game. Thegraphicswill be developedusing unrealengine 4 andZbrushwhich are bothprofessional industrytools andthusthegoal is to provide the game with high resolutiongraphics,capable of runningfrom $1080 \mathrm{p}$ to $360 \mathrm{p}$ screens. The game play does not require active internet connection to run and will be a single player storyline game play.

The software requirement for the development of this software is: I. UNREAL ENGINE 4 ii. BLENDER iii. ZBRUSH IV.AUTODESK MAYA v. GIMP VI. MIXAMO .The Current game system will be available as an exe installation to run on $\mathrm{PC}$ and the $\mathrm{PC}$ is required to possess a 
minimum requirement of:4GB Ram. $15 \mathrm{~GB}$ hard disk space. A Direct X 11 Graphic card (Nvidia or Geforce) Keyboard and Mouse or Game Joystick. The game will also be able to run on different resolutions according to the capabilities of the graphic card from $360 \mathrm{p}$ to $1080 \mathrm{p}$. There will be a user interface for arranging the setting of the game visual and game play and it will be up to the player to decide. Also there will be setting for the difficulty level for the game that will dynamically affect the game play.And the player can save and load their game progress and exit at will, a fully functional user interface is provided. We used Linear Sequential Model sometime it also called classical Life cycle or water flow model.Sequential model suggests a systematic sequential approach to software development that begins at the system level and progresses through the following.

Through research of the industry market, the action adventure genre is one of the highest demanding and grossing among the gamers. So the action adventure genre was chosen and the basic structure such as the assets and the components were decided and designed as a prototype. With the working title of Project Hysteria the development process begin.Unreal Engine 4 was the core component used as all the assets were arranged in it in a sequential order and an alignment of various assets and process were decided.After implementing all the component, the project was ready for testing. Our first objective in the testing phase will be the connectivity between the components on which the game is executing. The game went through various beta testing phase in which students and adults alike played the game and the first response was positive.Second testing phase was in collages and schools were students played the initial version and were curious to know when the first commercial version will be released for further experience of the game.

The project under creation is to provide a gaming revolution in Pakistan Fig1. Videogame industry is one of the highest grossing sectors in the technologicalfield [1].Withthedevelopmentofa game project, the aim is to motivate the aspiring gamedevelopersworkingonminorIndiegame projects, to take a major step and work on a major gametitleandthismayleadtointroductionof several game development teams and development studios [2]. We use one of the most powerful game enginesUnrealEngine4, whichisthecurrent version of the Unreal Game Engine series by Epic Games. With Unreal engine we use employ several powerful soft ware's such Autodesk Maya, Zbrush and Gimp.

Thegameisathird-personshootervideogame which goes on par with the graphically advanced games being released on current generation of the gaming consoles (Play Station 4 and Xbox One). Withtheprojectunderincrementaldevelopment and facing major changes and being continuously updated, upon release, it is certain to create the identity of Pakistan Game Industry in the international entertainment market andcompete with other current generation advance game titles.

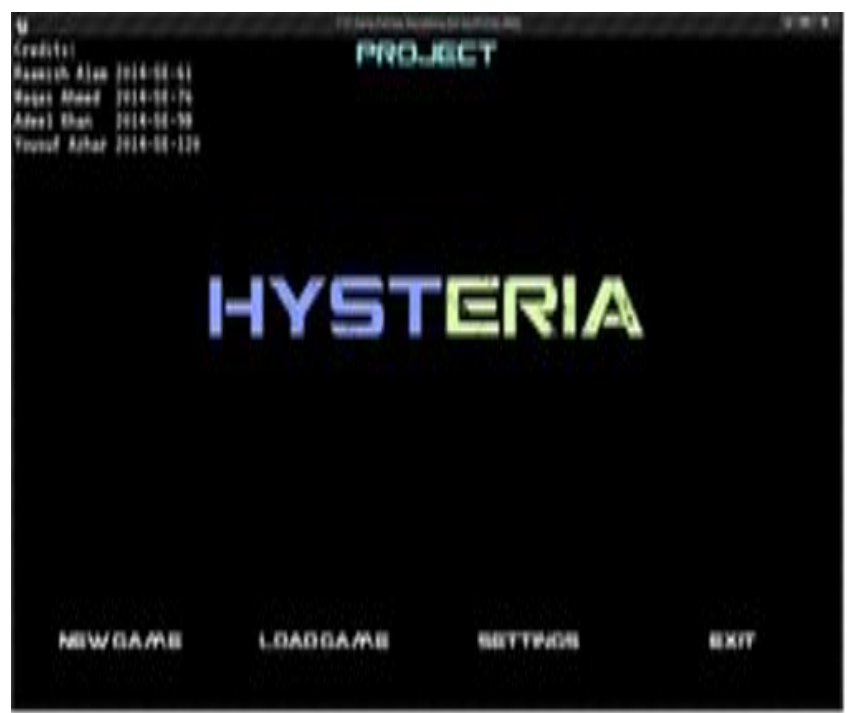

Fig. 1. The Main Title Screen of Hysteria Displaying All the Typical basic Options of a Title Screen.

\section{HYSTERIA STORYLINE}

There is now almost a tradition for a game of any genre to have an interesting and catchy storyline in order to captivate the attention of consumers. We also took the liberty of designing our original storyline.

Considering various factors such as survival, action, drama,adventureandheavysciencefiction,our story is set in a fictional universe in which human beings have reached out and conquer other planets and their reach have extended to outer space. The main character of our story Commander Anderson fig2. is a Galactic Patrol Federation commando whose space ship crashed in an unfamiliar planet of which the environment heavily resembles that of earth but much more exotic. This planet is HYST-51891 an unidentified planet is occupied by hostile and savage human beings whose origins are unknown. They arrived suddenly and drove the previous occupants to extinction.

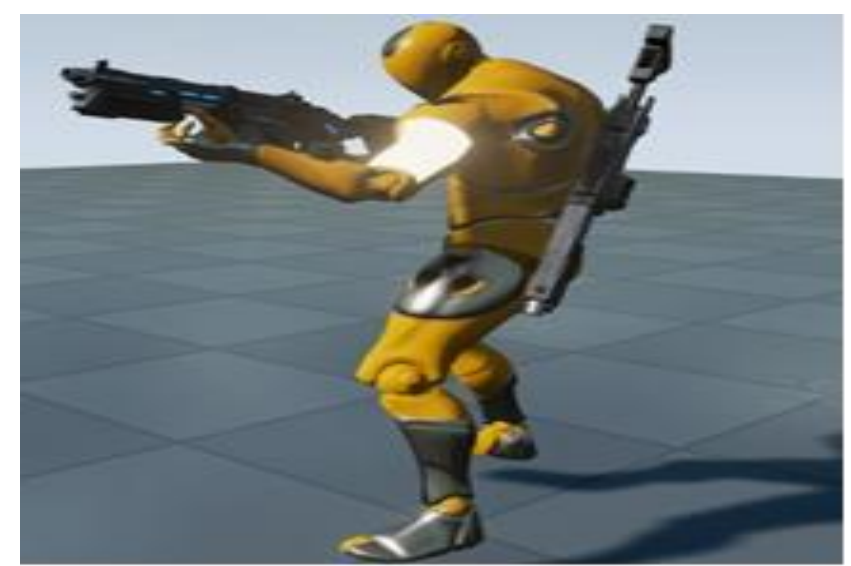

Fig. 2. Commander Anderson, The Main Character Donning the Galactic Patrol Federation Armor and Wielding a Laser Assault Rifle. 


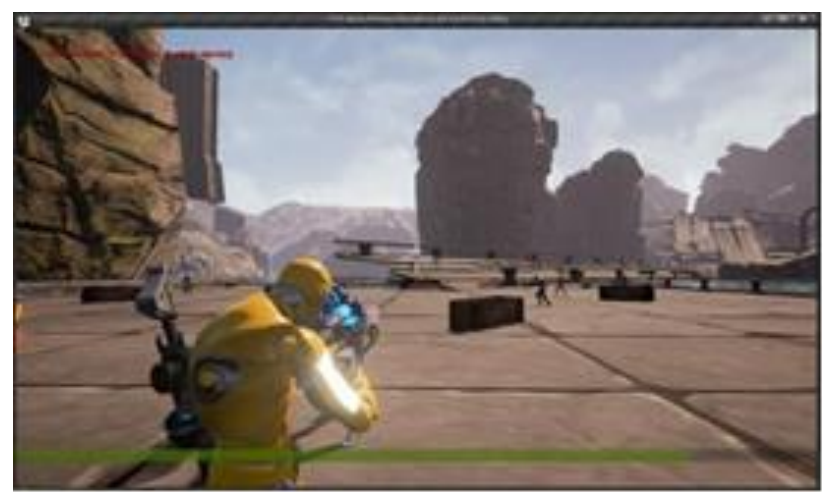

Fig. 3. The Game Play Screenshot.

On the planet Commander Anderson is attempting to fix his space ship or get any sort of help when he is ambushed by the hostile native of the planet fig3. These human beings are ruthless to intruders and wield the same advance armors and weaponry used by the Galactic Patrol Federation and are known as the Demons. He must know battle these creatures and escape the planet as the planets climatic changes are eventually drawing its catastrophic destruction. As he traverses through many environments from exotic green ruins to the deep jungle, from the frozen frost wastelands to the fire landsofHYST-51891alsoknownasHysteria's Hell, he must defeat all the enemies that cross his path and find a way to escape the impending doomsday on the planet.

The story is science fiction themed and manages to bestow upon the player a sense of survival as the number and fighting intensity of adversaries will grow as the player passes through each level.

\section{SOFTWARES USED IN DEVELOPING HYSTERIA}

Hysteria is a science fiction action adventure game played from a third person perspective in which the player must shoot the enemies and avoid enemy fire by using the cover objects. We used industry standard tools that are used by the modern game development studios to create professional commercial games. Some of them are listed below:

1) Unreal engine 4: There are a number of professional tools that we used to bring our vision to life, the most notable and the trump being the unreal engine 4 game engine which acts as a compiler which arranges each of the individual objects created sequentially and in proper order fig 4 . It is also used to give the color, textures and contrast of the environments and characters. While the assets of the game may be developed in other software unreal engine 4 is used to modify these assets in order to make them look realistic and beautiful [6]. It also provides the basic graphic options of scaling, translation and rotation which are heavily used for modifying and arranging the environment together.Scalingandtranslationis used to extend the platforms created using blenders whilerotationisusedtorotateandarrangethe scaled environment in perfect order.

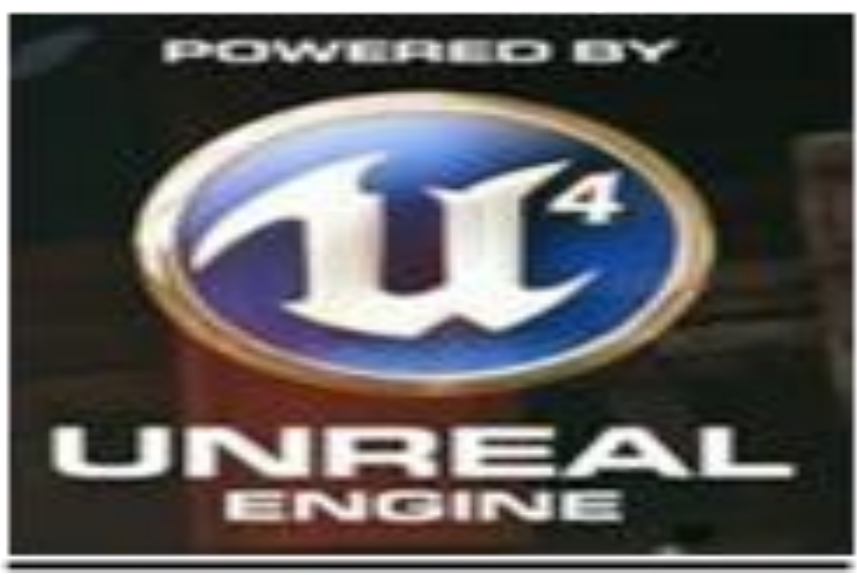

Fig. 4. Hysteria is Powered by Unreal Engine 4.

It is also used for giving the player and the enemies' characters skeletons. This is accomplished through the in game rendering. Unreal engine 4 has blueprint technology implement into it which can be used as a sort of visual coding [3]. It is important as it has been used to create the main artificial intelligence between the player characters.

2) Blender: Blender is an open-source 3D computer graphic software that is used for creating $3 \mathrm{~d}$ printed models used in our game [4] [11]. Many of our assets are that are used in game are created using blender. For e.g. the guns that are wielded by Commander Anderson and the enemies was modelled in blender and then exported to unreal engine 4 in which it was given propertextureandfinally implementedin on the joint point of the player and enemies skeleton to make an appearance that they are actually wielding the gun.

The blender is only used for modelling an object as itscolor;contrastandtexturearegiveninany graphicsoftwarethatitisimportedto,suchas Autodesk Maya, 3DsMax or in our case, the unreal engine 4 fig 5.

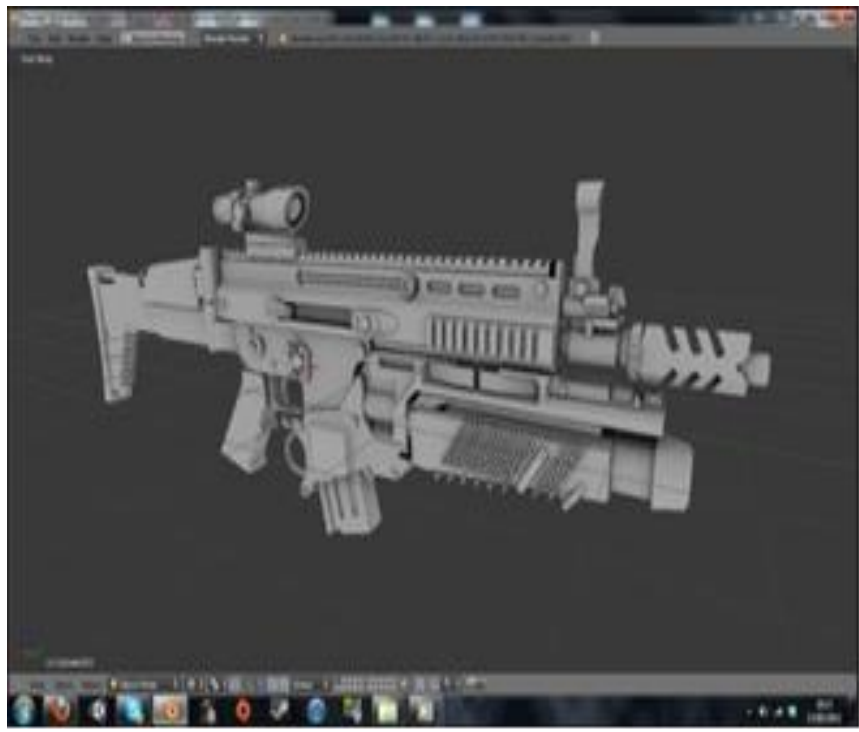

Fig. 5. The Assault Rifle Is Modelled using Blender and then Exported to Unreal Engine 4. 
3) Zbrush: Zbrush is used in the animation and gaming industry tocreatehighresolutionmodes(ableto reach40+million polygons) [5] . In our project, although it is used for a small role, it plays a critical small role as some of the contrast for enemies and player character and the environments (mountains, rocky terrai8n and statues) were modified using Zbrush.It is an advanced tool that could have been used to create even more detailed characters for our gamesbuttheideawasomittedwelackthe necessary time to modelthe charactersin such a complicated tool.

4) Autodesk maya: Unreal engine 4 consists of an A.R.Ts (Animation and Rigging Tools) which is used for animating and rigging in game characters using Autodesk Maya [7]. This plug in of Autodesk Maya possess a default character rig model which is used to create animation (character moving and running) fig 6 . These animationscan be exported to unreal engine 4 to test in game play and can be replaced by our default model.

This is optional as the Unreal Engine 4 also has the in game rendering system but the downside is that it is of inferior quality than the A.R.Ts. That why A.R.Tsis usedby mostanimatorsas it not only eases up the animation process but have far more versatile options while animating characters.

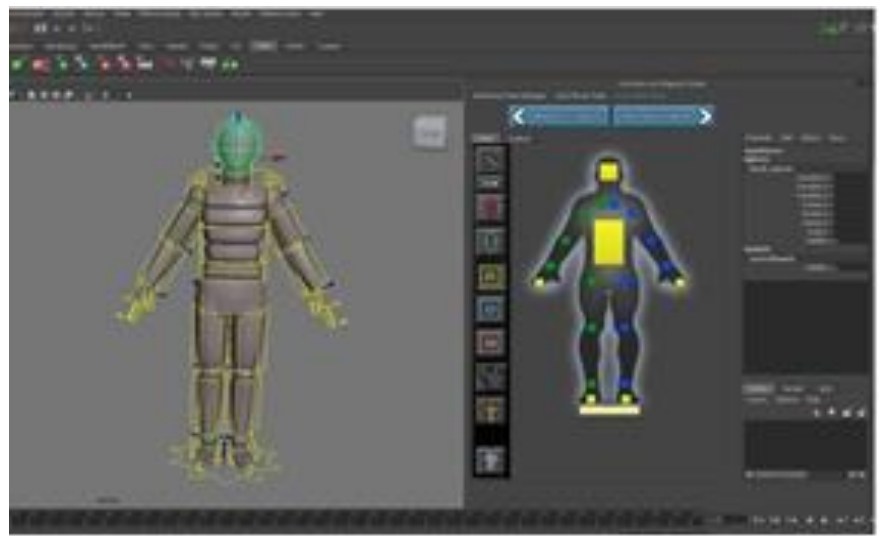

Fig. 6. The Unreal Engine 4 A.R.Ts Plug in Running on Autodesk Maya.

\section{DEVElOPMENT OF HYSTERIA}

1) Artificial intelligence: The artificial intelligence is the most crucial part of our game. It is how the player and enemy react to each other [8]. The enemies have been programmed with $\mathrm{AI}$ to react in certain situations such as:

- To attack the player when in their line of sight.

- To petrol an area under which they are stationed.

- To become aware of the player character when hit by player fire and the player is not in their line of sight.

- To keep firing at the player until the player character runs out of health/ life bar.

- To cease fire once the player dies.

- To take cover when under player fire.
- To use assault rifle when fighting player.

- To use shotgun when fighting player.

- To use sniper rifle while taking cover using cover object when fighting player.

- To lay down dead when their health / life bar runs out (Death animation).

The following AI situations have been programmed using the blueprint technology of the unreal engine 4 . It is its visual scripting system.

As shown in Fig 7, the blueprint programming consists of various nodes that are arranged and scripted to make the characters react to certain situations. The nodes as shown in the above diagram are connected together to make an enemy do certain things. It is basically programming their reaction and their default behavior, for e.g. in An Enemy blueprint, there will be a node for enemy to be in stationary position and that exact node extends to patrolling a certain area and that node will extend to move to one waypoint and then move to the other waypoints andthenback,bygoingbackand forwardto the waypoints,a patrolof the areais beingcompleted,andiftheplayercharacteris enemy preprogrammed sight, there is a node that will engage the enemy character with the player in gun fight and as their health bar runs out, there will be a node of death animation for the enemy.

The player character will have similar nodes but most of their actions will be consisted in the node which gives player the overall control of the player character.

The nodes have to be pre-programmed by the developer and when certain actions and reactions are programmed in to the node, there is virtual artificial intelligence within game [10].

The Fig 8. also gives a clearer example as there is node arrangement in the player character session as there is a node arrangement for updating the camera view of the game as the player progress forward using the L key. Notice how the nodes are all connected together so that when the player makes the player character move, the camera will be programmed to automatically follows.

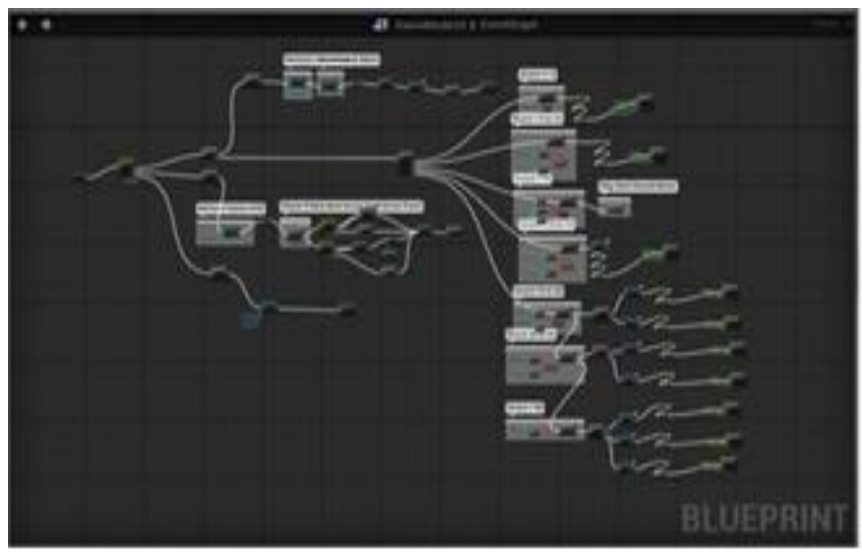

Fig. 7. The Blueprint Technology is used to Orchestrate the in Game Artificial Intelligence. 


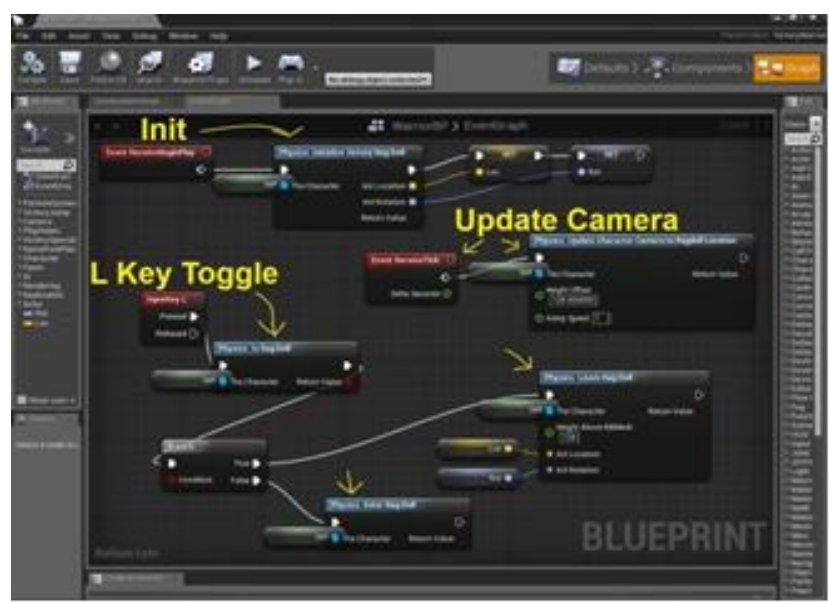

Fig. 8. Nodes of Camera, Character and Key Toggle.

Hence blueprint technology is one of the most important features of the unreal engine 4 as it is directly responsible for planning down and developing the in game functionalities and sequencing [9].

2) Environment creation: The environment in game was developed using unreal engine 4 and blender. Blender was first platforms on which the player and enemy character move fig 9. It was used to create objects that were later on programmed to avoid enemy fire and hence became covers.

The platforms are exported into the unreal engine 4 andmanipulated byusingtheingame functionalities of scaling (the platforms were extended in order to create the floor and ground), translation (use to transit the scale floor and grounds to proper place) for creating mountains and rotation was used to rotate the objects into proper order fig 10. Besides the usual movement of these manipulated objectsinto properplace, unrealenginewasalso

usedtogivethosecontras, texturesandoverall realistic feelings.

Blender is also used heavily in creating the hallway levels of the game as the platform were heavily modified and arranged together (using scaling, translation and rotation) with blender to give it an appearance of the hallway, only after arranging the hallway together was it exported into the unreal engine to give it contrast and textures.

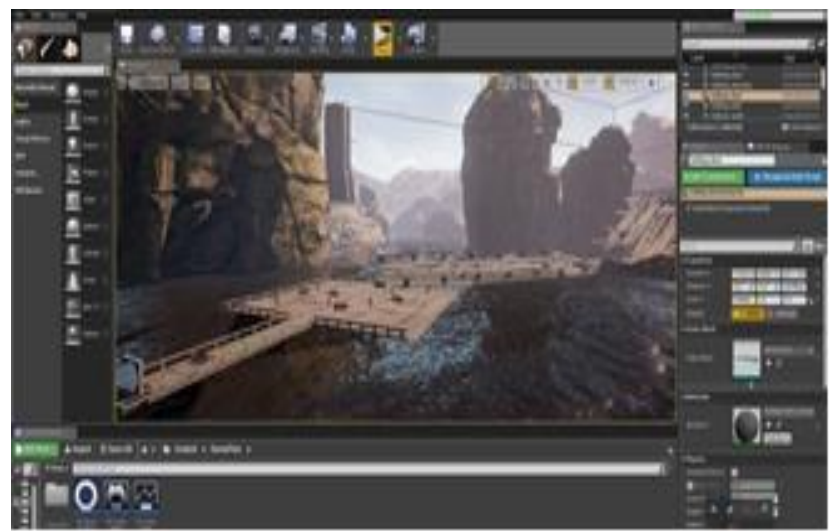

Fig. 9. Environment Manipulation of Scaling To Create Extended Floor from Platforms.

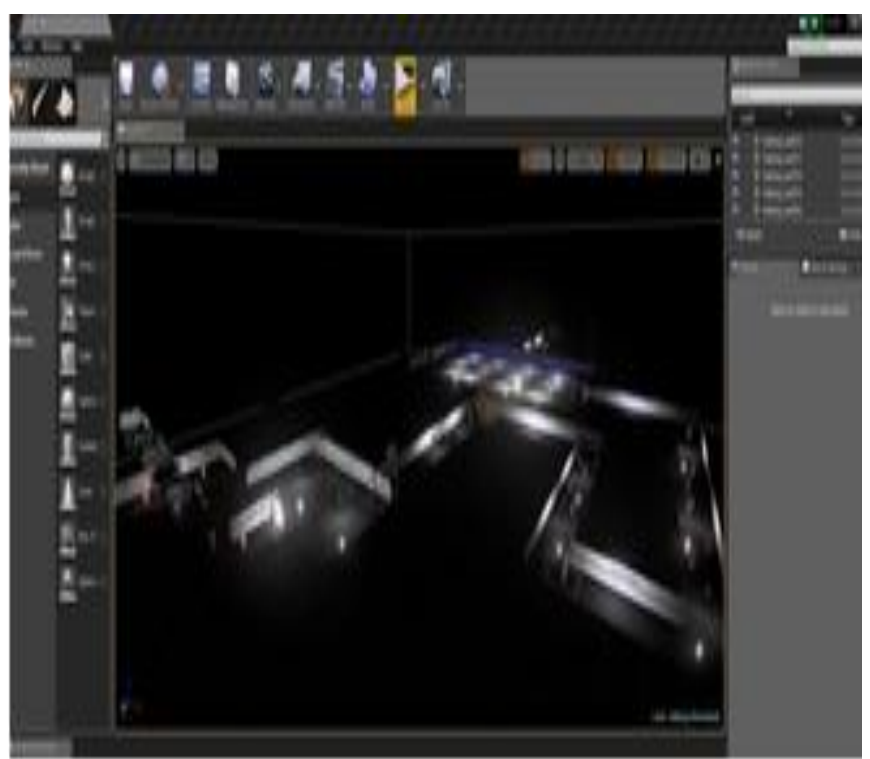

Fig. 10. The Hallway Arranged through Many Scaled, Translated and Rotated Platforms in Unreal Engine 4 with Contrast.

The unreal engine 4 is the most recent version of the unreal game engine by Epic Games. Due to it developed for making games on next generation consoles(8th generationgames)itssuperior graphics is credited for the realistic look of the environmentas it is used to developgame using high end graphic cards fig 11 . Only graphic cards with a direct $\times 11$ is appropriate for developing a game in unreal engine 4 or playing a game title developed in unreal engine 4.

Some of the environments consist objects such as trees and statues that are too difficult and complicated for contrasting and texturing in unreal engine 4 , this is why software like Zbrush can be appropriate for such job fig 12 .

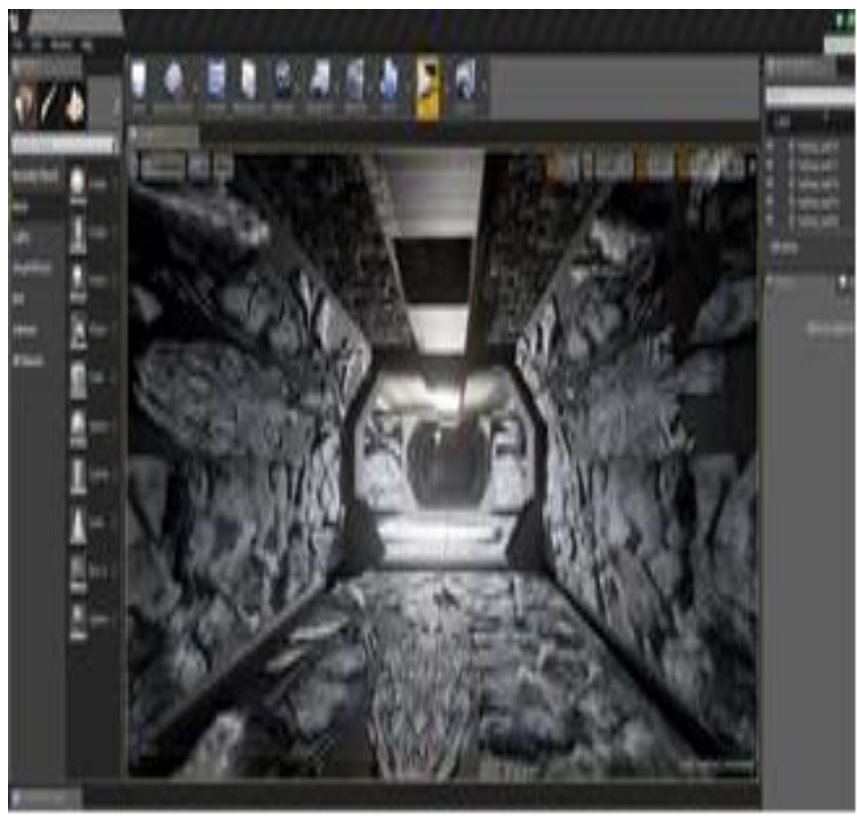

Fig. 11. The Hallway after giving the Frozen Wastelands Contrast, Notice the Superior Graphics. 


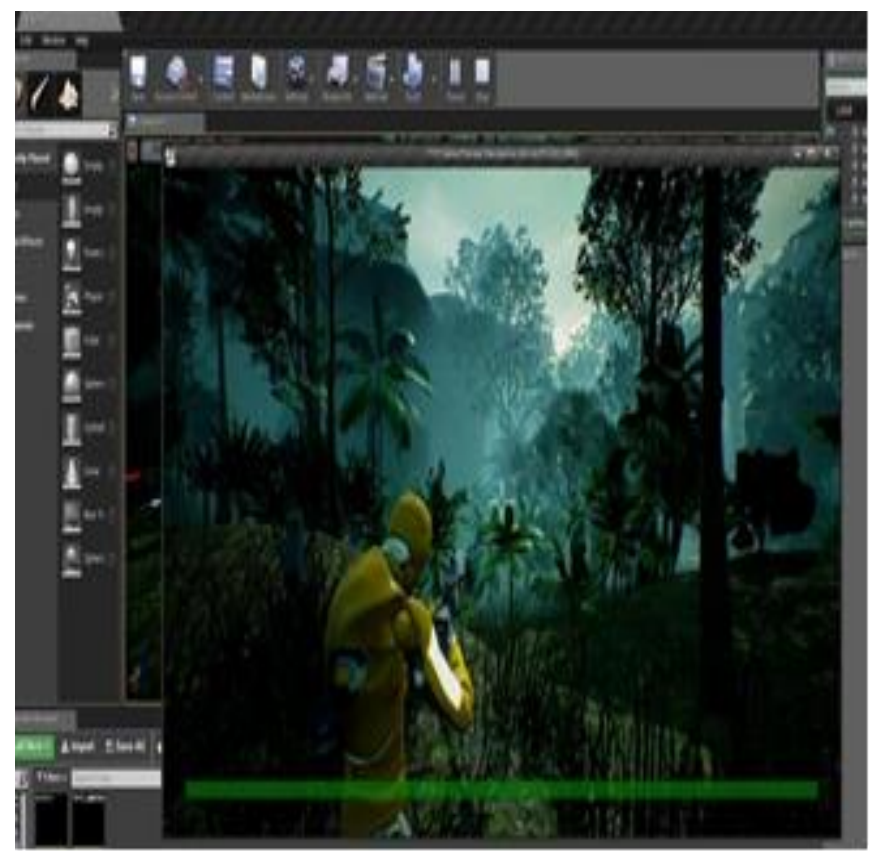

Fig. 12. The in Development Screenshot, the Trees are Textured in Zbrush.

3) Character creation: The characters and enemies all follow same character skeleton render and are contrasted and textured using unreal engine 4 and Zbrush.

They are programmed to wield both one assault rifle and one shotgun or one sniper rifle.

Their artificial intelligence programmed with unreal engine 4 blueprints allows them to move, attack each other, and take cover when under enemy fire (either player with player character or enemy with enemy AI) fig 13.

\section{CONCLUSION}

With the release of one of the first major studio console game title HYSTERIA from Pakistan, an era of gaming revolution is to be inspired. Many of the indie-game developers will be inspired to create creative major game titles in advanced game engines such as Unreal Engine and Cry Engine and thedevelopmentofPakistanigamestudiosown game engine will become in-evitable and this may leadtomajorgamingrevenuetobegenerated within Pakistan. More advance games with artificial intelligence implementation can also be developed for business prospective.

\section{FUTURE WORK}

In future more advance version of (HYSTERIA) game expected with latest graphics tools such as Unity 3D.This game introduced new trend of game development using Unreal engine 4 in game industry of Pakistan. Artificial intelligence can also provide new ways in field of game revolution.

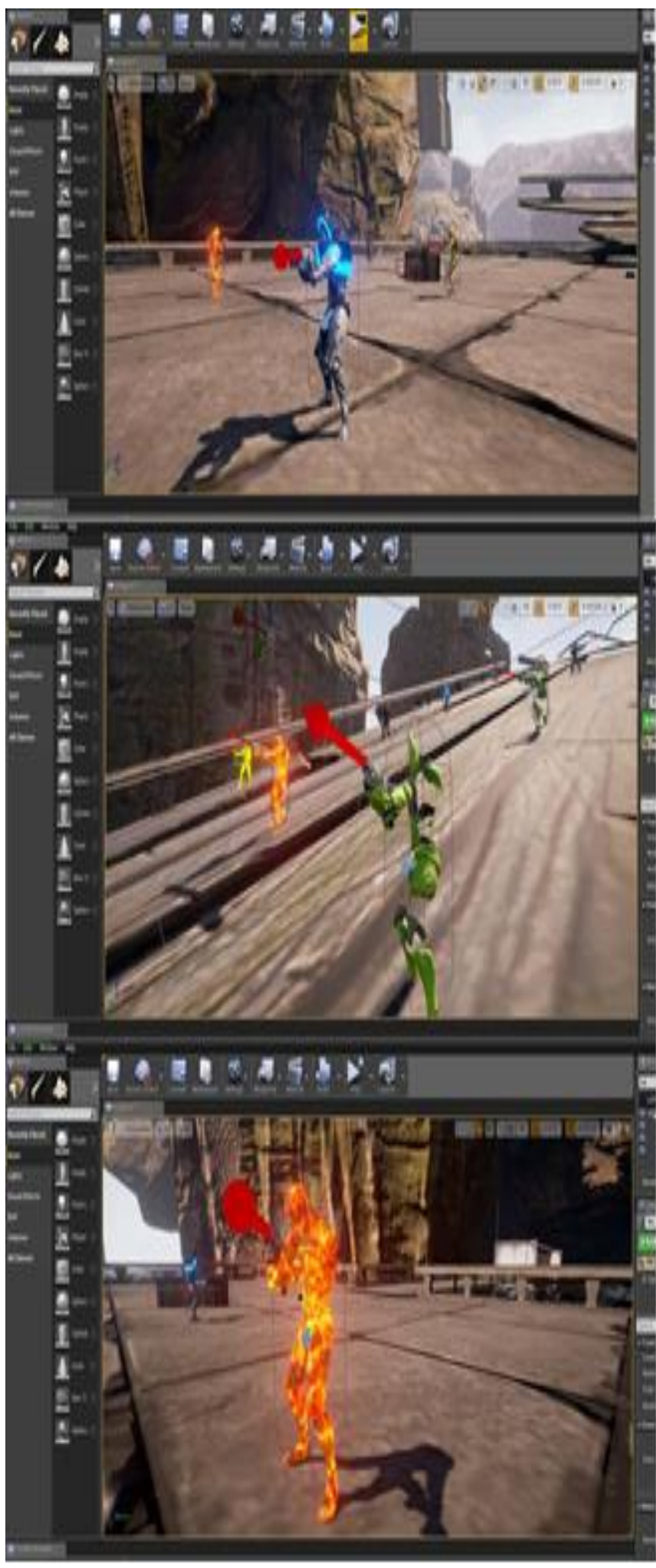

Fig. 13. Some of the Enemy Characters in Development View Window of the Game. 


\section{REFERENCES}

[1] Zackariasson and t. L. Wilson (eds). "The video game industry: formation, present state, and future", published book, June 8, 2012

[2] The observation that "indie games" as a movement within game development are a contemporary phenomenon has been made before (for example, see Lipkin, 2013, p. 9)

[3] Brian Tayler, "Introduction to Unreal Engine 4, 2014.

[4] BLENDER, "Open Source 3D creation. Free to use for any purpose, forever”, (https://www.blender.org/), Retrieved 15 Aug. 2018

[5] Zbrush Manual, www.Pixelogic.com, Retrieved May 20, 2008.
[6] Unreal Engine Mechanics, www.epicgames.com.Retrieved July 10,2018

[7] Todd Palmer, "Mastering AutoDesk Maya", Book published by Autodesk official press (2016)

[8] Arc Sosangyo. "Artificial Intelligence: The Most Important Technology for Our Future". Updated on May 24, 2018,

[9] Kevin Smith and Andersonbadk, "Blue print technology in Unreal Engine, 2015.

[10] Peter L. Newton and Jie Feng,. "Unreal Engine 4 AI Programming Essentials" June 6, 2016

[11] Justin Plowman., “ 3D Game Design with Unreal Engine 4 and Blender", June 29, 2016 